Proc. of the 15th Int. Workshop on Slow Positron Beam Techniques and Applications, Prague, September 2-6, 2019

\title{
Simulation of Positronium Decays in View of Charge Conjugation Symmetry Test with the J-PET Detector
}

\author{
J. ChHokar* on Behalf of the J-PET Collaboration \\ Faculty of Physics, Astronomy and Applied Computer Science, Jagiellonian University, 30-348 Cracow, Poland

\begin{abstract}
We present search of the C-forbidden decays of positronium with the J-PET detector. J-PET is a first tomograph based on plastic scintillators and, due to large acceptance and high angular resolution, it is suitable for studies of various phenomena such as: discrete symmetries in decays of positronium atom or entangled states of photons as well as the medical imaging. In view of the C-symmetry test, the J-PET is used inter alia to determine the angular distribution of the three annihilation photons from positronium decay.
\end{abstract}

DOI: 10.12693/APhysPolA.137.134

PACS/topics: 78.70.Bj, 41.75.Fr, 36.10.Dr

\section{Introduction}

In 1918, a celebrated theorem connecting invariance of a system with respect to continuous global transformation and a corresponding conserved quantity was proven by Amalie Emmy Noether [1], for example: translation in time, space or rotation conserves energy, momentum, or angular momentum, respectively. In case of discrete symmetries (such as reflection in space $P$, reversal in time $T$, and charge conjugation $C$ ) Noether's theorem is not applicable. However, these symmetries appear to be conserved in the process driven by the gravitational, electromagnetic, and strong interactions but were experimentally shown to be violated in weak interactions. Symmetries have become crucial part of modern physics and retained the interest of scientific community in pursuing the explanation of inequality between the matter and anti-matter in the universe. According to Sakharov [2] and Fukugita and Yanagida [3] the asymmetry as we see requires violation of the $C$ and $C P$ symmetries at a level that cannot be explained so far with known phenomena. Thus, there is still a lot of interest in finding new sources of nonconservation for these symmetries, particularly in the positronium decays.

This article is arranged as follows: first, we explain the $C$-symmetry selection criteria for the annihilation of positronium and the principle of operation of the Jagiellonian Positron Emission Tomograph (J-PET) including the techniques and software, used to analyse the data. Further on, we present the simulated results for the allowed $3 \gamma$ decay $\left({ }^{3} S_{1} \rightarrow 3 \gamma\right)$ from positronium.

*corresponding author; e-mail: jyotichhokar48@gmail.com

\section{Charge conjugation symmetry in decays of positronium}

Charge conjugation is a transformation associated with the exchange of particles and antiparticles by changing the signs of all additive quantum numbers (for example, electric charge). Positronium (Ps) is built out of electron and anti-electron and thus it is the simplest object to study the charge conjugation symmetry $[4,5]$. Positronium in the ground state (orbital angular momentum $l=0)$ can be formed as a spin singlet state known as para-positronium (p-Ps: ${ }^{1} S_{0}$ ) with antiparallel spins orientation, or in a triplet state known as ortho-positronium (o-Ps: ${ }^{3} S_{1}$ ). The general selection rule for the annihilation of Ps from a state of orbital angular momentum $l$ and total spin $s$ into $n$ photons is given by [6]:

$$
(-1)^{l+s}=(-1)^{n} \text {. }
$$

Photons are $C$-odd and thus $C$ eigenvalue of $n$ photons system is equal to $(-1)^{n}$, whereas the $C$ operator acting at Ps interchanges the electron and positron, yielding a factor of $(-1)^{l+s}$, since they have opposite intrinsic parity. Conservation of symmetry under charge conjugation forbids annihilation of p-Ps into an odd number of photons and o-Ps annihilating into an even number of photons. The simplest $C$-forbidden decay of positronium atom is the singlet state $\left({ }^{1} S_{0}\right)$ annihilating into 3 photons.

This annihilation mode $\left({ }^{1} S_{0} \rightarrow 3 \gamma\right)$ was studied experimentally by Mills and Berko [7] in 1967. Considering Bose statistics $C$-nonconserving ${ }^{1} S_{0} \rightarrow 3 \gamma$ rate must vanish for the case of the three photons emerging symmetrically in the Ps centre of mass frame (photons emission relative angles equal to $120^{\circ}, 120^{\circ}, 120^{\circ}$ ), independently of the assumed form of the $C$-nonconserving interaction [8]. Mills and Berko studied the count rate at three different angular configurations of three photons. They estimated the branching ratio $R$ of ${ }^{1} S_{0} \rightarrow 3 \gamma$ decays $3 \gamma / 2 \gamma$ with best limit so far $\left(R \sim 2.6 \times 10^{-6}\right.$ at $68 \%$ confidence level [7]). 


\section{Test on $C$ symmetry using the J-PET detector}

J-PET, as a large acceptance detector, is built out of 192 strips of polymer scintillators (EJ-230) arranged axially in three cylindrical layers [9]. The dimension of each scintillator is $1.9 \mathrm{~cm} \times 0.7 \mathrm{~cm} \times 50.0 \mathrm{~cm}$ with the longest side of scintillator arranged along $z$-axis as shown in Fig. 1. The tomograph enables investigations of positronium atoms in medical applications $[10,11]$. However, its superior time resolution (about 100 ps [12]), high granularity of detection strips and lower detection pile-ups provides new research opportunity for discrete symmetries [5] and quantum entanglement [13, 14].

Photons from $\mathrm{e}^{+} \mathrm{e}^{-}$annihilation interact with the plastic scintillator strips, and usually as a result of scintillation, a few thousands of photons are emitted isotropically for $511 \mathrm{keV}$ gamma quanta. Photomultipliers (PMTs, Hamamatsu R9800 vacuum tube) are placed at opposite ends of each strip and convert these scintillation light into electrical signals which are read out by multi-threshold digital electronics [15] and the data are continuously collected by trigger-less and re-configurable data acquisition system [16, 17]. Each analog signal from the PMT is sampled in the voltage domain at four thresholds. Probing signals allows to reconstruct the original shape of the signal. To process and analyse the measured data from experiment and simulation, a dedicated offline framework has been developed $[18,19]$ which is highly flexible, ROOT-based software package which aids reconstruction and calibration procedure for the tomograph. Characterized by high angular resolution (of about $1^{\circ}$ ) along with coverage of almost whole phase space (as discussed in Sect. 3.2) and with better timing properties (about 100 ps resolution), J-PET stands out to be well-suited for the study of charge conjugation violation in search of three photons from the decay of para-positronium.

\subsection{Simulation of the 3 gamma events at J-PET}

Simulations presented in this paper were performed using the Geant-4 package which controls the tracking of particles through detector geometry and uses well tested routines to simulate interactions. While the description of the J-PET detector and details on the oPs $\rightarrow 3 \gamma$ annihilation process are incorporated as a part of the package.

Events were simulated in the centre of the detector (as shown in left part of Fig. 2), assuming the isotropic emission from the point-like ${ }^{22} \mathrm{Na}$ radioactive source. One event in this case consists of 3 hits which are 3 annihilating photons from the decaying o-Ps. These photons are coplanar in the center of mass due to momentum conservation and based on the position of source and points of interactions, we can determine the relative angles between annihilated photons as we can see in Fig. 2. For studies presented in this paper $10^{9}$ events of ortho-positronium decaying into three photons were simulated.
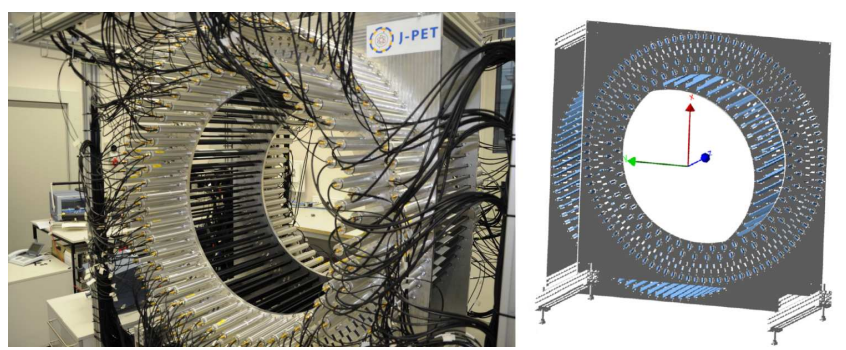

Fig. 1. (left) Photograph of the J-PET detector consisting of 192 plastic scintillators arranged (along $z$ axis) in three layers. (right) Simulated geometry of the J-PET scanner with metal frame holding scintillators (blue). Simulation were performed using Geant-4 package.
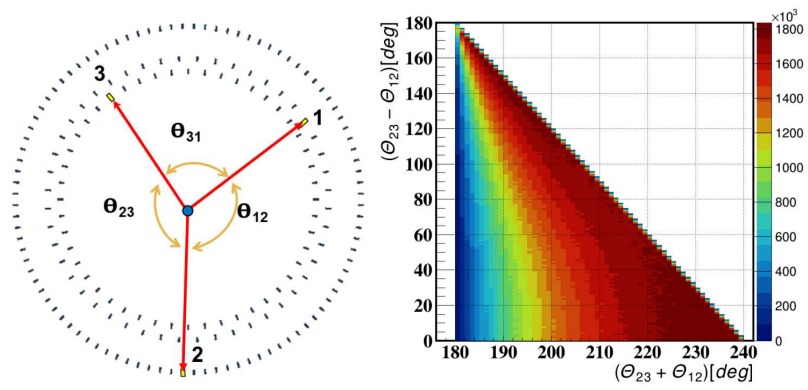

Fig. 2. (left) Schematic representation of the simulated $3 \gamma$ events where $\Theta_{12}, \Theta_{23}$, and $\Theta_{31}$ represents the relative angle between first, second, and third photons annihilating from o-Ps and (right) the relation between the sum and difference of two smallest relative angles $\left(\Theta_{12}\right.$ and $\left.\Theta_{23}\right)$ of the three interacting annihilation photons.
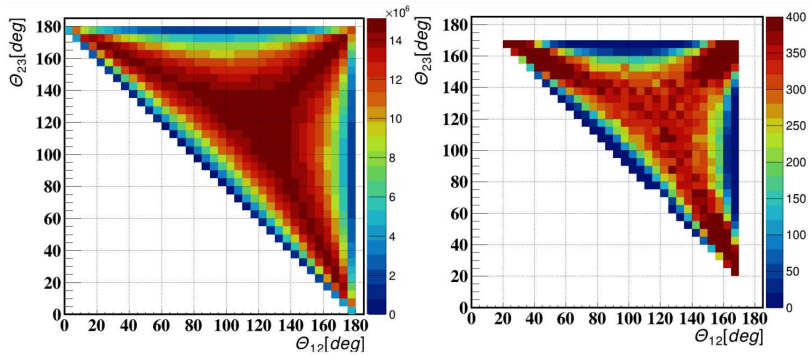

Fig. 3. Distribution of relative angle $\Theta_{12}$ and $\Theta_{23}$ of o-Ps $\rightarrow 3 \gamma$ annihilation, for the true generated decays (left) and corresponding reconstructed simulations (right).

The conducted simulations accounted for the angular distribution of the emitted photons in the J-PET detector, and further reconstruction of hit-time and hitposition of the registered gamma interaction in the detector has been simulated taking into account experimentally determined resolutions. Based on hit position and hit time of each generated and reconstructed event, 


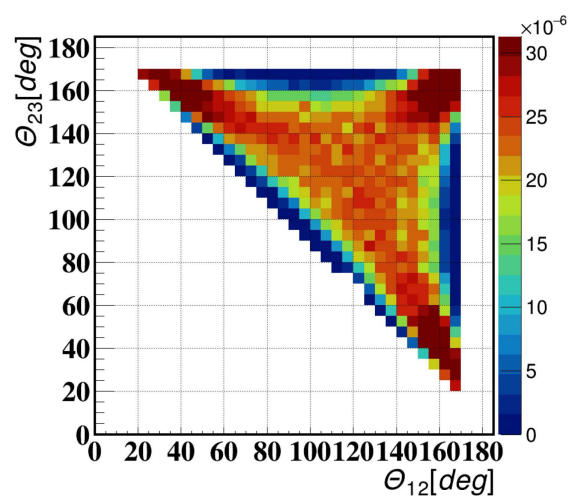

Fig. 4. Efficiency map of the J-PET detector as a function of $\Theta_{12}$ and $\Theta_{23}$ relative angles obtained by dividing bin-by-bin spectra for generated and reconstructed simulations showed in Fig. 3.

momentum of each photon is calculated and based on the momenta, relative angles between the three photons are determined. Using angles between annihilation photons we obtained angular distributions for the simulated events (left part of Fig. 3) and for simulations after reconstruction (Fig. 3, right), due to the selection criteria some events are discarded in the reconstructed spectra. In particular, in order to suppress events from p-Ps $\rightarrow 2 \gamma$ with back-to-back photons (such events will be present in the real experimental data) we require that $\Theta_{i j}$ is less than $170^{\circ}$. Dividing the reconstructed and generated distributions allowed to obtain an efficiency map for the studied ortho-positronium decay, as shown in Fig. 4.

\section{Conclusions and perspectives}

We have performed realistic Monte Carlo simulations (using Geant-4 package) of the detection performance of the Ps $\rightarrow 3 \gamma$ decay with the J-PET detector, which shows that J-PET enables to study the charge conjugation symmetry violation via $\mathrm{p}-\mathrm{Ps} \rightarrow 3 \gamma$ decay. Analysis of the simulations leads to determination of the J-PET efficiency map. Produced result presented in this article shows that due to almost whole coverage of allowed phase space and relatively high detection efficiency J-PET detector is a suitable tool to measure three photon decays.

Now we are planning to perform analogous simulations for $C$-non conserving process of p-Ps decaying into 3 photons and to obtain experimentally angular distribution of 3 photons originating from o-Ps and p-Ps decays. In order to separate the allowed and forbidden decays to 3 gamma quanta we plan to use the distribution of positronium lifetime [20]. Due to a difference in a lifetimes we can clearly differentiate between para- (lifetime of $125 \mathrm{ps}$ ) and ortho-positronium (lifetime in XAD4 of about $90.8 \mathrm{~ns}$ ). Our current focus is to compare angular spectra of 3 photons decay for short and long lived positronia and validate them against simulated data in order to identify forbidden p-Ps $\rightarrow 3 \gamma$ decay.

\section{Acknowledgments}

The authors acknowledge the support by the Foundation for Polish Science through the MPD and TEAM POIR.04.04.00-00-4204/17/ programmes, the National Science Centre of Poland through grants no. 2016/21/B/ST2/01222, 2017/25/N/NZ1/ 00861, the Ministry for Science and Higher Education through grants no. 6673/IA/SP/ 2016, 7150/E$338 / \operatorname{SPUB} / 2017 / 1$.

\section{References}

[1] A.E. Noether, Nachr. d. Gesellsch. d. Wiss. zu Göttingen Math.-Phys. Klasse 2, 235 (1918) (in German); [English translation: Transp. Theory Statist. Phys. 1, 186 (1971)].

[2] A.D. Sakharov, Pisma Zh. Eksp. Teor. Fiz. 5, 32 (1967).

[3] M. Fukugita, T. Yanagida, Phys. Lett. B 174, 45 (1986).

[4] M. Deutsch, Phys. Rev. 82, 455 (1951).

[5] P. Moskal, D. Alfs, T. Bednarski, et al., Acta Phys. Pol. B 47, 509 (2016).

[6] S. Berko, H.N. Pendleton, Ann. Rev. Nucl. Part. Sci. 30, 543 (1980).

[7] A.P. Mills, S. Berko, Phys. Rev. Lett. 18, 420 (1967).

[8] D.C. Liu, W.K. Roberts, Phys. Lett. 16, 67 (1966).

[9] P. Moskal, O. Rundel, D. Alfs, et al., Phys. Med. Biol. 61, 2025 (2016).

[10] P. Moskal, D. Kisielewska, C. Curceanu, et al., Phys. Med. Biol. 64, 055017 (2019).

[11] P. Moskal, B. Jasińska, E.Ł. Stępień, S.D. Bass, Nature Rev. Phys. 1, 527 (2019).

[12] P. Moskal, Sz. Niedźwiecki, T. Bednarski, et al., Nucl. Instrum. Methods Phys. Res. A 764, 317 (2014).

[13] B.C. Hiesmayr, P. Moskal, Sci. Rep. 7, 15349 (2017).

[14] B.C. Hiesmayr, P. Moskal, Sci. Rep. 9, 8166 (2019).

[15] M. Pałka, P. Strzempek, G. Korcyl, et al., J. Instrum. 12, P08001 (2017).

[16] G. Korcyl, D. Alfs, T. Bednarski, et al., Acta Phys. Pol. B 47, 491 (2016).

[17] G. Korcyl, P. Białas, C. Curceanu, et al., IEEE Trans. Med. Imag. 37, 11 (2018).

[18] W. Krzemień, M. Bała, T. Bednarski, et al., Nukleonika 60, 745 (2015.

[19] A. Wieczorek, P. Moskal, S. Niedźwiecki, et al., Acta Phys. Pol. A 127, 1491 (2015).

[20] K. Dulski, C. Curceanu, E. Czerwiński, et al., Hyperfine Interact. 239, 1 (2018). 